\title{
Molecular theory of the tilting transition and computer simulations of the tilted lamellar phase of rod-coil diblock copolymers
}

\author{
M.A. Osipov ${ }^{1,2}$, M.V. Gorkunov ${ }^{3}$, A.V.Berezkin ${ }^{2}$, A.A. Antonov ${ }^{2,3}$ and Y.V.Kudryavtsev ${ }^{2,41}$ \\ ${ }^{1}$ Department of Mathematics and Statistics, University of Strathclyde, Glasgow G1 1XH, Scotland, \\ $U K$ \\ ${ }^{2}$ Topchiev Institute of Petrochemical Synthesis, Russian Academy of Sciences, 119991 Moscow, \\ Russia \\ ${ }^{3}$ Shubnikov Institute of Crystallography, Federal Scientific Research Centre "Crystallography and Photonics", \\ Russian Academy of Sciences, 119333 Moscow, Russia \\ ${ }^{4}$ Frumkin Institute of Physical Chemistry and Electrochemistry, Russian Academy of Sciences, 119071 Moscow, \\ Russia
}

(Dated: 19 February 2020)

Symmetric rod-coil diblock copolymers have been simulated using the method of dissipative particle dynamics in the broad range of the Flory-Huggins parameter. It has been found that the tilted lamellar phase appears to be the most stable one at strong segregation. The profiles of the tilt angle and the orientational order parameters have been determined as functions of the segregation strength. The density functional theory of rod-coil diblock copolymers has been generalised to the case of the tilted lamellar phase and used to study the stability of the orthogonal lamellar phase with respect to tilt. The orthogonal phase indeed appears to be unstable in the broad region of the parameter space in the case of relatively strong segregation. It has also been shown that he transition into the tilted lamellar phase is determined by a strong coupling between two independent tilt order parameters.

\section{INTRODUCTION}

Rod-coil block copolymers attract significant attention both from the fundamental and from the application point of view because they are interesting soft matter materials which combine the properties of coil-coil (flexible) block copolymers and those of liquid crystals including, in particular, the orientational order of rod-like fragments. Rod-coil copolymers are composed of macromolecules which contain a rigid and a flexible parts linked together. Rod and coil monomers are incompatible, but the bond between the rod-like fragment and the coil prevents the system from macroscopic phase separation. The rod blocks can be semiconducting polymers ${ }^{1,2}$, polypeptides $^{3,4}$ and polysaccharides ${ }^{5,6}$ which leads to a number of applications in polymer photovoltaics ${ }^{1,7,8}$, LEDs $^{9-11}$, and high strength polymer composites ${ }^{12,13}$.

The self-assembly or rods and coils in rod-coil copolymers is different from that in coil-coil block copolymers due to the conformational asymmetry between rigid and flexible blocks and the anisotropic interactions between rods which result in a strong orientational order. Rod-coil block copolymer melts exhibit a number of microphase separated and orientationally ordered phases including the nematic, orthogonal, tilted and perforated lamellar, hexagonal columnar, bicontinuous and body-centred cubic and wavy lamellar or zigzag phases (see, for example, the reviews ${ }^{14-16}$ ). One notes that some of these phase are very similar to the corresponding anisotropic phases exhibited by low molecular weight liquid crystals. In particular, the orthogonal lamellar phase possesses exactly the same symmetry as the smectic A phase and the tilted lamellar phase is similar to the smectic $\mathrm{C}$ phase. On the other hand, the hexagonal columnar phase, the orthogonal lamellar and some cubic phase are also found in coil-coil block copolymers.

The properties of rod-coil block copolymers are not completely understood theoretically although a number of dif- ferent approaches have been used to describe these systems. The first statistical theory of rod-coil block copolymers has been developed by Semenov and Vasilenko who have employed a simple lattice model with the perfect orientational order of rigid rods ${ }^{17}$. A more sophisticated theory has been developed using the Landau-de Gennes expansion of the free energy in terms of the equilibrium densities of rod and coil monomers and the orientational order parameter or rigid rods ${ }^{18,19}$. The coefficients of such a free energy expansion have been calculated using Flory-Higgins theory ${ }^{18}$ or employing the monomer-monomer correlation functions of the system of noninteracting copolymer chains ${ }^{19}$ calculated following the approach proposed by Leibler ${ }^{20}$. The theory based on the free energy expansion has been used to describe a number of different phases including the nematic, smectic $\mathrm{C}$, orthogonal lamellar, hexagonal and body-centred cubic phase ${ }^{19}$. At the same time such an approach is generally valid only in the case of weak segregation or close to the transition into the disordered phase where the equilibrium densities are approximately proportional to the corresponding order parameters. The theory also does not describe the orientational order of rigid rods in the lamellar and the hexagonal phase which is observed experimentally ${ }^{14}$.

The self-consistent field theory (SCFT), which has been very successful in the description of the coil-coil block copolymers, has also been applied to rod-coil block copolymers $^{21-25}$. In this theory the free energy of a single chain is calculated by numerical evaluation of the path integral along the chain while the interaction between monomers in different chains is taken into account in the random phase approximation. One notes, however, that it is difficult to simulate three dimensional polymer systems with orientational degrees of freedom and anisotropic interchain interactions as this leads to the dramatic increase of the computational cost. As a result in the past the SCFT theory has been applied to 
rod-coil block copolymers using simplified models including lattice models ${ }^{23,24}$, two dimensional models ${ }^{22}$, or the models with perfect orientational order ${ }^{21,23,24}$. Recently, a massive parallel three- dimensional SCFT scheme has been applied to a 3D rod-coil diblock copolymer with a Gaussian chain by Kriksin and Khalatur ${ }^{25}$ who have been able to reveal the chiral columnar phase composed of nonchiral macromolecules.

In another version of the SCFT theory the evaluation of the path integral along the chain is replaced by solving the modified diffusion equations for semiflexible chains ${ }^{26-34}$. This theory has been even more successful in the description of rodcoil block copolymers as it enables one to describe the variety of different phases and to obtain some information about the orientational ordering of rigid chains. Recently it has been shown ${ }^{28-31,35}$ that the modern SCFT theory enables one to describe 3D systems in the general case. For example, a number phase diagrams have been obtained which contain lamellae, hexagonal, gyroid and cubic phase ${ }^{28}$. The nematic phase and the orthogonal and tilted lamellae phases have been described by Song et.al. ${ }^{29}$, and the effect of rigidity of a semiflexible chains on the phase behaviour of rod-coil block copolymers with worm like chains has been studied in et.al. ${ }^{31}$. In this approach, however, both the flexible block and the rigid block are modelled using the same formalism of flexible chains characterised by different persistence lengths.

Recently the classical density functional approach, which is successfully used in the molecular theory of liquid crystals $^{36-40}$ and inhomogeneous fluids ${ }^{41}$, has been applied by the authors to rod-coil diblock copolymers ${ }^{42}$. In the density functional approach the free energy is expressed as a functional of the equilibrium densities of rod and coil monomers which are obtained by minimization of the free energy functional. The free energy also depends on the direct correlation functions between rod and coil monomers in the reference system of noninteracting copolymer chains. The molecular theory ${ }^{42}$ is based on the self-consistency equations for the translational and orientational order parameters and does not employ the expansion in terms of the order parameters. Thus it is valid in the case of relatively strong segregation and at low temperatures when the equilibrium monomer densities are nonlinear functions of the order parameters containing many Fourier harmonics. The theory enables one to calculate numerically the orientational and translational order parameters in the lamellar phase in an efficient way.

So far there have been few computer simulation studies of rod-coil diblock copolymers ${ }^{43-47}$. For example, dissipative particle dynamics simulations of discrete rigid rods linked to spheres connected via the harmonic potential in an isothermalisobaric ensemble have revealed the isotropic, smectic A and crystalline phases ${ }^{46}$. In the molecular dynamics simulations of Wilson et.al. ${ }^{43}$ in an isothermal isobaric ensemble the rigid block has been modelled by a spherocylinder and the coil was represented by a sequence of tangential spheres. In this study the isotropic, nematic and smectic A phases have been found. Finally Glotzer et al. have used Brownian dynamics simulations in a canonical ensemble and found a number of interesting morphologies ${ }^{44,47}$.

We focus into the properties of the tilted lamellar phase.
This phase has been first described by Semenov ${ }^{48}$ in the framework of his lattice theory ${ }^{17}$ with perfect orientational order. The model of Semenov has later been used in the SCFT theory ${ }^{21}$ but the approximation of the perfect orientational order has been preserved. Recently the smectic $\mathrm{C}$ phase has been obtained in the framework of the modern SCFT theory based on the model of wormlike chains ${ }^{29,31}$. According to ref. ${ }^{29}$ the orientational order parameter of rods in the smectic C phase is very high and the tilt angle decreases with the increasing parameter $\chi$. In Ref. ${ }^{31}$ it has been shown that the rod-coil diblock copolymer undergoes a transition from the orthogonal into the tilted lamellae phase with the increasing parameter $\chi$ and the Mair-Saupe interaction constant. Very recently the tilted phase has also been described by Cai et al. ${ }^{32}$ who have investigated the structure and phase diagrams of bilayer membranes self-assembled from rod-coil diblock copolymers dissolved in a flexible homopolymer. Thus the stable smectic $\mathrm{C}$ phase has successfully been described by the existing theory in several cases. At the same time the microscopic mechanism of the tilting transition in the lamellar phase is still to be investigated.

In this paper, the density functional theory of rod-coil diblock copolymers is developed to describe the loss of stability of the orthogonal lamellar phase with respect to the director tilt. The mechanism of the transition into the tilted lamellar phase appears to be rather nontrivial as the orthogonal phase loses its stability simultaneously with respect to the two independent order parameters associated with the tilt. We have also undertaken the dissipative particle dynamics (DPD) simulations of rod-coil block copolymers and have found that the tilted lamellar phase is more stable then the orthogonal phase in the whole range of parameters investigated. We have also extracted the profiles of the tilt angle from simulations using the general theory of the smectic A - smectic C transition in low molecular weight liquid crystals developed by two of the authors $^{38}$.

The paper is arranged as follows. In Section II we discuss the simulation technique, present the results of the DPD simulations of the tilted lamellar phase including the order parameter and tilt angle profiles extracted from the simulation data. In Section III we present the general density functional theory of rod-coil diblock copolymers and in Section IV we consider the direct correlation functions between rod and coil monomers within one chain and a relationship with the corresponding density-density correlation functions. Section V contains the final expressions for the free energy density of the tilted phase and the results of the numerical calculations which are used to analyse the stability of the orthogonal phase with respect to tilt. Finally, Section VI contains the discussion and our conclusions. 


\section{COMPUTER SIMULATIONS OF ROD-COIL DIBLOCK COPOLYMERS}

\section{A. Dissipative particle dynamics}

During the past decades, DPD method has become very popular in soft matter simulations as a powerful tool to search for the microstructures in various block copolymer systems (see, for example, Ref. ${ }^{49}$ and references therein). DPD is a coarse-grained molecular dynamics technique proposed by Hoogerbrugge and Koelman ${ }^{50,51}$ for the simulation of liquid suspensions and extended to polymer systems by Espanol, Groot and Warren ${ }^{52,53}$ by mapping it onto the classical FloryHuggins theory.

A periodic simulation box of the size $l_{x} \times l_{y} \times l_{z}=32 \times 32 \times$ $32 r_{c}^{3}$ (where $r_{c}$ is a certain cut-off radius which is treated as a unit length ) is filled with a total of 98304 DPD particles of a rod-coil copolymer. A diblock copolymer chain $A_{x} B_{y}$ consists of $N=20$ bonded particles forming rigid A block and chainlike B block of lengths $N_{A}$ and $N_{B}$ respectively. Copolymer composition $N_{A} / N$ may vary, but this study is mainly focused on symmetric copolymer, where $N_{A}=N_{B}=10$.

The particles are soft and interact via conservative, dissipative, and random forces which are pairwise additive. The net force $\mathbf{F}_{i}=\sum_{j}\left(\mathbf{F}_{i j}^{C}+\mathbf{F}_{i j}^{D}+\mathbf{F}_{i j}^{R}\right)$ acting on a given particle $i$ is calculated as a sum over the forces from all other particles within the cut-off radius $r_{c}$. The conservative force represents the excluded volume interactions and elastic interactions of particles $i$ and $j$ in the dimensionless form $\mathbf{F}_{i j}^{C}=a_{i j}\left(1-r_{i j}\right) \hat{\mathbf{r}}_{i j}-k_{s} \mathbf{r}_{i j}$ where $\mathbf{r}_{i j}=\mathbf{r}_{i} \mathbf{r}_{j}, r_{i j}=\left|\mathbf{r}_{i j}\right|, \hat{\mathbf{r}}_{i j}=$ $\mathbf{r}_{i j} / r_{i j}, a_{i j}$ is a maximum repulsion between the particles located at $\mathbf{r}_{i}=\mathbf{r}_{j}$ and $k_{s}$ is a spring constant which is taken to be $k_{s}=4$ for particles linked in a polymer chain. The dissipative and random forces, $\mathbf{F}_{i j}^{D}=-\gamma \omega\left(r_{i j}\right)^{2}\left(\hat{\mathbf{r}}_{i j} \cdot \mathbf{v}_{i j}\right) \hat{\mathbf{r}}_{i j}$ and $\mathbf{F}_{i j}^{R}=\sigma \omega\left(r_{i j}\right) \xi(\delta t)^{-1 / 2} \hat{\mathbf{r}}_{i j}$, respectively, constitute the Groot-Warren thermostat ${ }^{53}$, where $\gamma$ is a friction coefficient related to a thermal noise amplitude $\sigma$ via the fluctuationdissipation theorem, $\gamma^{2}=2 \sigma, \omega(r)$ is a weight function, $\xi$ is a normally distributed random variable with zero mean and unit variance, which is uncorrelated for different particle pairs, $\delta t$ is the time step of an integration scheme, and $\mathbf{r}_{i j}=\mathbf{r}_{i}-\mathbf{r}_{j}$ is the relative velocity of particles $i$ and $j$. Following Ref. ${ }^{53}$, we choose $\sigma=3$ and $\omega(r)=1-r$.

The equations of particle motion, $d \mathbf{r}_{i} / d t=\mathbf{v}_{i}, d \mathbf{v}_{i} / d t=\mathbf{F}_{i}$ are solved numerically using a free source code LAMMPS ${ }^{54}$ that implements the modified velocity-Verlet algorithm (the so called DPD-VV integration scheme $)^{55}$ with a time step $\delta t=0.04$. In this section we define the unit time $\tau_{0}=$ $r_{c}\left(m / k_{B} T\right)^{1 / 2}$ where $r_{c}$, the particle mass $m$, and $k_{B} T$ as set as the unit distance, mass, and thermal energy, respectively.

The repulsion parameter between similar particles of a DPD liquid, $a_{\alpha \alpha}=25$, as recommended in Ref. ${ }^{53}$ at the chosen particle density $\rho_{0}=3$. It follows from our recent simulations ${ }^{56}$ and the existing literature that rigid ${ }^{46}$ and semi-rigid ${ }^{57}$ rods reveal a noticeable order at $N_{R}>7$. In this work we analyse longer rod-like blocks, which are very well ordered under these conditions.

The particles of the rigid blocks are connected by bonds of the constant length $b_{R}=0.7 r_{c}$. Nanorods are simulated as rigid bodies in the $N V E$ ensemble using an algorithm by Miller et al. ${ }^{58}$. Spring-like bond potentials do not apply in this case. Correct temperature of blocks $\mathrm{A}$ is maintained through the interactions with the B blocks, which are thermostated as in the conventional DPD.

To equilibrate the system, we start with the long relaxation during $2 \times 10^{6}$ time steps with the repulsion parameter $a_{A B}=$ 25. Then the simulated annealing is performed via a stepwise elevation of the repulsion parameter between different blocks, followed by the relaxation and the productive run (at every new value of $a_{A B}$ ). Duration of the relaxation period and the productive run are both equal to $5 \times 10^{5}$ each. Repulsion parameter increases in 20 steps with the increment of $\Delta a_{A B}=$ 2.0 up to the value of $a_{A B}=65$. Such a simulation covers the range of the segregation parameter $\chi N$ from 0 to 122.4 .

\section{B. Simulation data processing}

DPD simulations enable one to visualize stationary states of the composite and to describe them in terms of the local volume fractions $\phi_{A}(r)$ and $\phi_{B}(r)$. The scalar orientational order parameter, $S(r)$, that characterizes the average orientation of nanorod axes $\mathbf{a},(|\mathbf{a}|=1)$ relatively to the unit vector $\mathbf{k}$ normal to lamellae planes:

$$
S(\mathbf{r})=\left\langle\frac{3}{2}[\mathbf{a}(\mathbf{r}) \cdot \mathbf{k}]^{2}-\frac{1}{2}\right\rangle,
$$

where the angular brackets denote the averaging over a local subset of rods. Zero value of the order parameter corresponds to an uncorrelated orientation of nanorods, whereas $S>0(S<0)$ indicates their tendency to perpendicular (parallel) orientation with respect to the lamellar plane. Coordinate system of a single lamellar is shown in Fig. 1.

When a lamellar microstructure is formed, we are interested in the distribution of all components along the normal to the layers. However, the orientation of lamellae in a periodic simulation box is random and a regular procedure for extracting the desired distribution is needed. We make use of the fact that a normal to the lamellar plane can be defined as an average vector connecting the centre of masses of the block B with the junction point between blocks A and B in one copolymer chain. Such connecting vectors are plotted for all copolymers in the system, then normalized to the unit length and translated so that their starting points coincide with the origin of coordinates. Ends of those vectors now form a cloud of points $\mathbf{p}_{i}=\left(p_{i x}, p_{i y}, p_{i z}\right)$ non-uniformly distributed over the surface of a unit sphere. The gyration tensor of this cloud $J_{\alpha \beta}=(1 / n) \sum_{i=1}^{n} p_{i \alpha} p_{i \beta}$ is diagonalized to find three eigenvalues and eigenvectors. The eigenvector corresponding to the largest eigenvalue is the very vector $\mathbf{k}$ which is normal to the lamellar planes. Below we select the $z$-axis in the direction of the vector $\mathbf{k}$. All local variables which describe the copolymer structures are expressed as functions of $z: \phi_{A}(z)$, 
$\phi_{B}(z), \phi_{A}(z), S(z)$, while the while the $B / A$ interface is chosen as the origin $(z=0)$.

It should be noted that the tilted lamellar phaser is biaxial and hence the orientational order of rod fragments is characterised by two order parameters: the uniaxial order parameter $S$ and the biaxial order parameter $P$ similar to the liquid crystal smectic $\mathrm{C}$ phase The tensor order parameter is then expressed as:

$$
Q_{i j}=S\left(n_{i} n_{j}-\frac{1}{3} \delta_{i j}\right)+P\left(m_{i} m_{j}-h_{i} h_{j}\right)
$$

where $\mathbf{n}$ is the director, the unit vector $\mathbf{m} \perp \mathbf{n}$ is in the tilt plane and the unit vector $\mathbf{h}$ is normal to the tilt plane. The tilt angle is defined by the equation $\cos \theta=(\mathbf{n} \cdot \mathbf{k})$. One notes that it is rather difficult to extract the values of the tilt angle directly from the simulation data because the tilt angle cannot be rigorously expressed as a thermal average of some microscopic quantity.

The same tensor order parameter can also be expressed in another frame based on the layer normal $\mathbf{k}$ and the unit vector $\mathbf{c} \perp \mathbf{k}$ in the tilt plane which is parallel to the plane ${ }^{38}$ :

$$
Q_{i j}=S_{k}\left(k_{i} k_{j}-\delta_{i j} / 3\right)+\frac{1}{2} P_{k}\left(c_{i} c_{j}-h_{i} h_{j}\right)+\frac{1}{2} V\left(k_{i} c_{j}+c_{i} k_{j}\right),
$$

Here the order parameter $S_{k}$ characterizes the ordering of long molecular axes along the smectic layer normal $\mathbf{k}$. The order parameter $P_{k}$ is the nematic tensor biaxiality, i.e., it describes biaxial distribution of long molecular axes within the layer plane. Finally, the tilt order parameter $V$ is a coefficient in the non-diagonal term of the $\mathbf{Q}$ tensor and hence it specifies the tilt of its main axis with respect to the layer normal k. The order parameters $S_{k}, P_{k}$ and $V$ can be explicitly expressed as the following thermal averages of the corresponding molecular quantities:

$$
\begin{gathered}
S_{k}=\left\langle P_{2}(\cos \gamma)\right\rangle, P_{k}=\left\langle\sin ^{2} \gamma \cos 2 \varphi\right\rangle, \\
V=\langle\sin 2 \gamma \cos \varphi\rangle,
\end{gathered}
$$

where the angles $\gamma$ and $\varphi$ are shown in Fig. 1. Thus the transition into the tilted lamellar phase is signified by the emergence of the new order parameter $V$ which is described as a thermal average of the corresponding microscopic quantity and hence it can be measured directly.

The order parameters $S$ and $P$ and the tilt angle $\theta$ can be expressed in terms of the parameters $S_{k}, P_{k}$ and $V$ by the following equations ${ }^{38}$ :

$$
\begin{array}{r}
\tan 2 \Theta=\frac{V}{S_{k}-0.5 P_{k}}, \\
S=\frac{1}{4} S_{k}+\frac{3}{8} P_{k}+\frac{3 V}{4 \sin 2 \Theta}, \\
P=\frac{1}{2} S_{k}+\frac{3}{4} P_{k}-\frac{V}{2 \sin 2 \Theta} .
\end{array}
$$

Thus it is possible first to evaluate the order parameters $S_{k}, P_{k}$ and $V$ directly from the simulation data and then to calculate the tilt angle from Eq. (6).

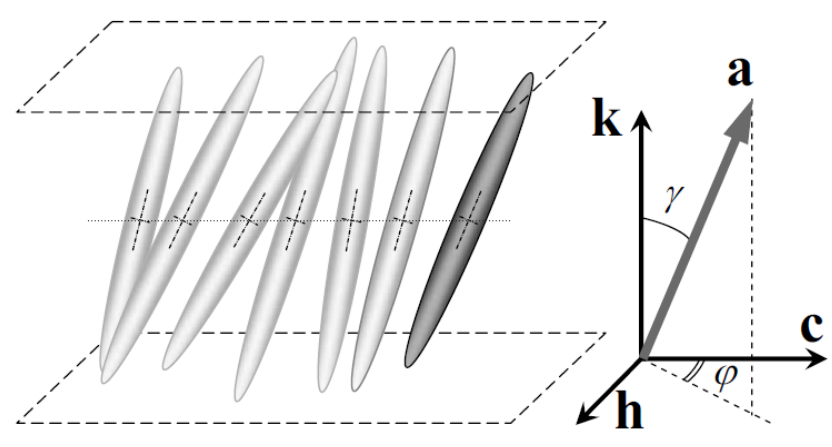

FIG. 1. Coordinate system related to the lamellar domains

Eqs. $(4,5)$ for the order parameters can be used directly if the orientation of the macroscopic tilt plane is known. The tilt plane is defined by the layer normal and the director $\mathbf{n}$ which has been found by the diagonalization of the inertia tensor $J_{i j}$, described above, with $\mathbf{p}_{i}=\mathbf{a}_{i}$ for each rod, where the unit vector $\mathbf{a}_{i}$ is in the direction of the rod axis. Then the coordinate system in Fig. 1 is completely defined and the parameters $P_{k}$ and $V$ can readily be found.

\section{Simulation results}

An accurate estimate of the order parameters is possible only for well-defined lamellar structures with no defects or dislocations at the scale of the domain size. The lamellar morphologies have been simulated for $N_{A}>8$. However, in the copolymers with $N_{A}=8$ and $N_{A}=9$ the lamellar stricture is characterised by some imperfections due to relatively high mobility of the chains and relatively weak ordering of rods. On the other hand, for $N_{A}>12$, the dislocations in the lamellar structure cannot relax during the time of the simulation due to extremely slow diffusion of long rods. The rod length $N_{A}=10$ which corresponds to the symmetric copolymer $A_{10} B_{10}$, appear to be optimal one for the analysis of the orientational ordering of the rods since in this case the defect-free lamellar morphologies are observed within the whole range of the block segregation parameter $\chi N$. The typical lamellar morphology arising at high segregation $(\chi N=97.9)$ is shown in Fig. 2.

The rigid A blocks are strongly orientationally ordered within each lamella. In this case the orientational order parameter $S$ mainly reflects the average angle between the layer normal $\mathbf{k}$ and the director $\mathbf{n}$. The average angle $\gamma$, calculated from the value of $S$ in the middle of A domain using Eq.(1), is presented in Fig. 3.

The order parameters $P_{k}$ and $V$ as functions of the coordinate $z$ are shown in Fig. 4. One can readily see that the order parameters are nearly constant within the lamella and drop at the edges, as one may expect. The values of these parameters at the centre of block $\mathrm{A}$ are shown in Fig. 5 as functions of the segregation strength $\chi N$. It is interesting to note that some biaxiality of the lamellar phase $P_{k}$ appears already at $\chi N=0$ 


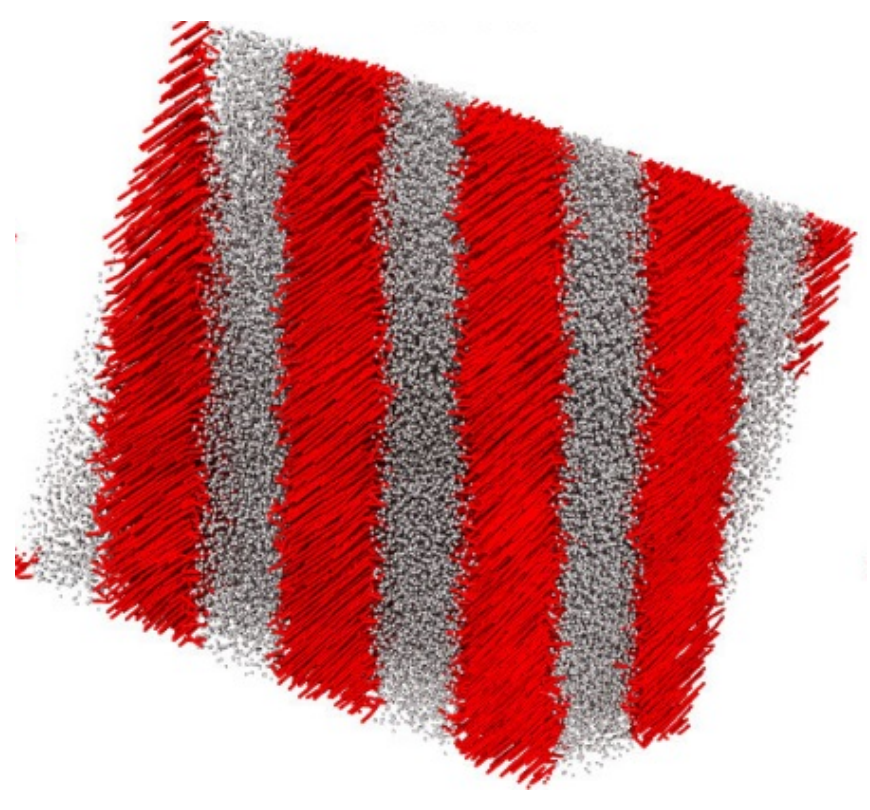

FIG. 2. Snapshot of the ordered $A_{10} B_{10}$ rod-like diblock copolymer at $\chi N=97.9$

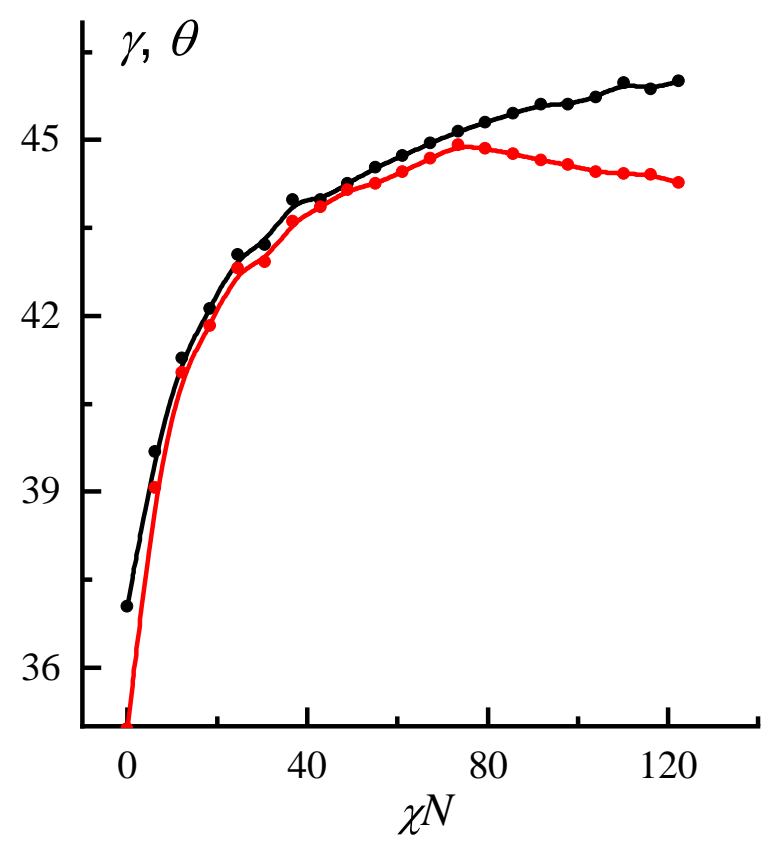

FIG. 3. Dependences of the tilt angle $\theta$ (red) and the average angle $\gamma$ (black) on the segregation parameter $\chi N$

and then grows proportionally to the segregation parameter $\chi N$. Simultaneously, the tilt in increasing as shown in Fig. 5

Finally it is possible to calculate the tilt angle $\theta$ from Eq. (6) using the values of $S_{k}, P_{k}$ and $V$ presented in Fig. 5 and to compare it with the effective average angle $\gamma$ calculated from the values of $S$. The results are presented in Fig. 3 where a good agreement is found until $\chi N=73.4$.

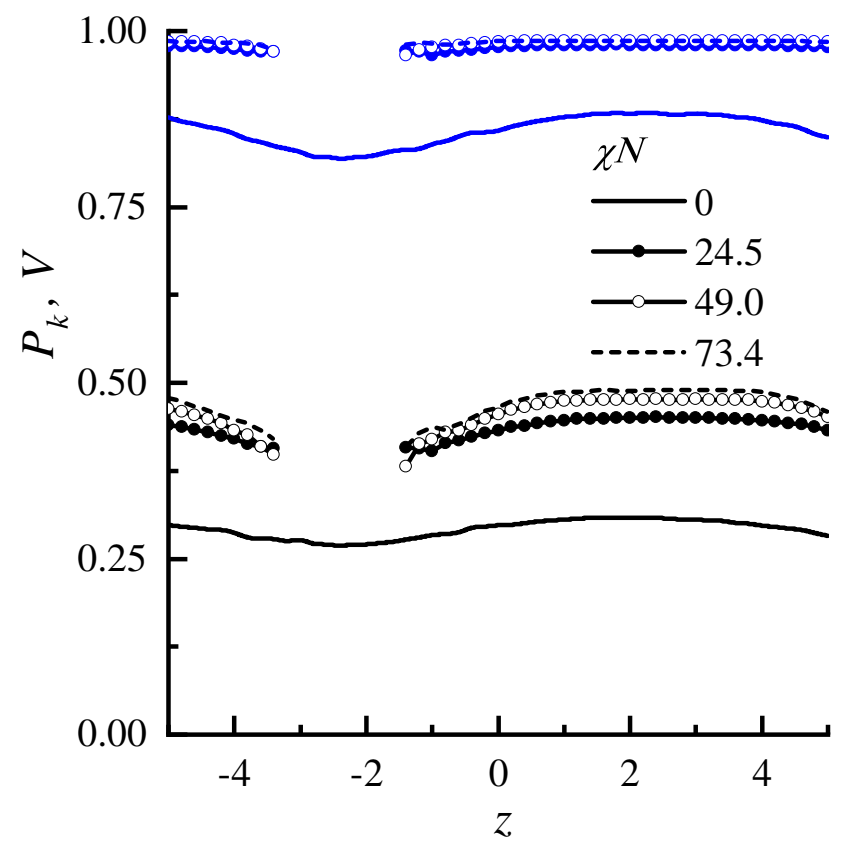

FIG. 4. Profiles of the order parameters $P_{k}$ (black) and $V$ (blue) across the lamella for different values of the segregation parameter $\chi N\left(N_{A}=N_{B}=10\right)$

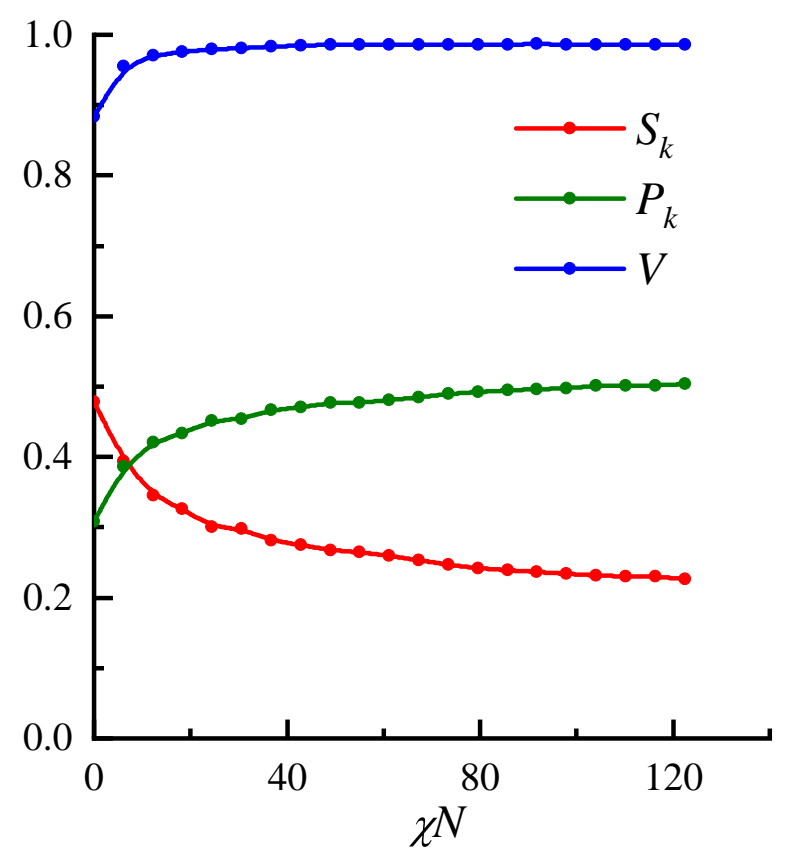

FIG. 5. Dependences of the order parameters $S_{k}, P_{k}$, and $V$ calculated in the middle of A domain on the segregation parameter $\chi N\left(N_{A}=\right.$ $\left.N_{B}=10\right)$ 


\section{DENSITY FUNCTIONAL THEORY OF ROD-COIL BLOCK COPOLYMERS}

A molecular-statistical theory of orientationally ordered rod-coil diblock copolymers can be derived in a general way using the density functional approach applied to the case of anisotropic fluids ${ }^{36-40,59,60}$. This approach has been successfully used in the molecular theory of liquid crystals ${ }^{36-39,59}$ and a gradient version of the theory has been applied to coil-coil block copolymers ${ }^{60}$. In this approach, the free energy $F$ of an anisotropic fluid is a functional of the one-particle density in the phase space $\rho(\mathbf{r}, \omega)$ which generally depends on the position $\mathbf{r}$ and orientation $\omega$ of the particles. The den- sity is normalised by the total number of particles $N$, i.e., $\rho(\mathbf{r}, \omega) d \mathbf{r} d \omega=N$. The density is proportional to the oneparticle distribution function $f(\mathbf{r}, \omega): \rho(\mathbf{r}, \omega)=N f(\mathbf{r}, \omega)$ as $\int f(\mathbf{r}, \omega) d \mathbf{r} d \omega=1$ The general structure of the free energy functional $F[\rho(\mathbf{r}, \omega)]$ is not known, but its functional derivatives are related to the direct correlation functions of the system. Then the free energy of the anisotropic phase can be obtained by performing a functional Taylor expansion of the free energy around its value in the isotropic phase.

Following the recently developed statistical theory ${ }^{42}$ the free energy functional of the rod-coil diblock copolymers can approximately be expressed as:

$$
\begin{aligned}
\beta F=\beta F_{I}+\int \rho_{v}(\mathbf{r}, \mathbf{a})\left[\ln \rho_{v}(\mathbf{r}, \mathbf{a})-1\right] d \mathbf{r}+\int \chi\left(r_{12}\right) \delta \rho_{r}\left(\mathbf{r}_{1}, \mathbf{a}_{1}\right) \delta \rho_{c}\left(\mathbf{r}_{2}, \mathbf{a}_{2}\right) d \mathbf{r}_{1} d \mathbf{r}_{2} d \mathbf{a}_{1} d \mathbf{a}_{2} \\
-\frac{1}{2} \int J\left(r_{12}\right) P_{2}\left(\mathbf{a}_{1} \cdot \mathbf{a}_{2}\right) \delta \rho_{r}\left(\mathbf{r}_{1}, \mathbf{a}_{1}\right) \delta \rho_{c}\left(\mathbf{r}_{2}, \mathbf{a}_{2}\right) d \mathbf{r}_{1} d \mathbf{r}_{2} d \mathbf{a}_{1} d \mathbf{a}_{2} \\
-\frac{1}{2} \sum_{v, \eta=r, c} \int C_{v, \eta}\left(\mathbf{r}_{12}, \mathbf{a}_{1}, \mathbf{a}_{2}\right) \delta \rho_{v}\left(\mathbf{r}_{1}, \mathbf{a}_{1}\right) \delta \rho_{\eta}\left(\mathbf{r}_{2}, \mathbf{a}_{2}\right) d \mathbf{r}_{1} d \mathbf{r}_{2} d \mathbf{a}_{1} d \mathbf{a}_{2}
\end{aligned}
$$

where $F_{I}$ is the free energy of the isotropic phase, $C_{c c}\left(\mathbf{r}_{12}\right)$, $C_{r c}\left(\mathbf{r}_{12}, \mathbf{a}_{1}, \mathbf{a}_{2}\right)$ and $C_{r r}\left(\mathbf{r}_{12}, \mathbf{a}_{1}, \mathbf{a}_{2}\right)$ are the coil-coil, rod-coil and rod-rod direct correlation functions calculated in the reference disordered phase of noninteracting copolymer chains, and $\delta \rho=\rho-\rho_{0}$ is the difference between the one-particle densities in the inhomogeneous phase and the density of the isotropic phase. Here the interchain interactions are taken into account in the molecular field approximation, including the repulsion between rod and coil monomers, specified by the Flory-Higgins parameter $\chi$, and the Maier-Saupe orientational interaction potential between rods $J\left(r_{12}\right) P_{2}\left(\mathbf{a}_{1} \cdot \mathbf{a}_{2}\right)$.

Minimization of the free energy (9) with respect to the number densities of rod and coil monomers yields the following self-consistent equations for $\rho_{c}\left(\mathbf{r}_{1}\right)$ and $\rho_{r}\left(\mathbf{r}_{1}, \mathbf{a}\right)$ :

$$
\begin{aligned}
\rho_{c}\left(\mathbf{r}_{1}\right)= & Z_{c}^{-1} \exp \left\{\int C_{c c}\left(r_{12}\right) \delta \rho_{c}\left(\mathbf{r}_{2}\right) d \mathbf{r}_{2}-\right. \\
& \left.-\int\left[\chi\left(\mathbf{r}_{12}\right)-C_{c r}\left(\mathbf{r}_{12}, \mathbf{a}\right)\right] \delta \rho_{r}\left(\mathbf{r}_{2}, \mathbf{a}\right) d \mathbf{r}_{2} d \mathbf{a}\right\} \\
\rho_{r}\left(\mathbf{r}_{1}, \mathbf{a}\right)= & Z_{r}^{-1} \exp \left\{\int C_{r r}\left(\mathbf{r}_{12}, \mathbf{a}\right) \delta \rho_{r}\left(\mathbf{r}_{2}, \mathbf{a}\right) d \mathbf{r}_{2}-\right. \\
& -\int\left[\chi\left(\mathbf{r}_{12}\right)-C_{c r}\left(\mathbf{r}_{12}, \mathbf{a}\right)\right] \delta \rho_{c}\left(\mathbf{r}_{2}\right) d \mathbf{r}_{2}+ \\
& \left.+\beta \int J\left(\mathbf{r}_{12}\right) P_{2}\left(\mathbf{a} \cdot \mathbf{a}_{2}\right) \delta \rho_{r}\left(\mathbf{r}_{2}, \mathbf{a}_{2}\right) d \mathbf{r}_{2} d \mathbf{a}_{2}\right\}
\end{aligned}
$$

where $Z_{c}$ and $Z_{r}$ are the corresponding normalization factors.

\section{DIRECT CORRELATION FUNCTIONS OF ROD-COIL DIBLOCK COPOLYMERS}

It is important to establish a relationship between the direct correlation functions of diblock copolymers and the corresponding density-density correlation functions which can be calculated analytically in the case of long polymer chains. The direct correlation function of single component system is defined by the well known Ornstein-Zernike equation:

$$
\begin{aligned}
& g_{2}\left(\mathbf{x}_{1}, \mathbf{x}_{2}\right)=C_{2}\left(\mathbf{x}_{1}, \mathbf{x}_{2}\right)+ \\
& \qquad C_{2}\left(\mathbf{x}_{1}, \mathbf{x}_{3}\right) g_{2}\left(\mathbf{x}_{3}, \mathbf{x}_{2}\right) \rho\left(\mathbf{x}_{3}\right) d \mathbf{x}_{3},
\end{aligned}
$$

where $\mathbf{x}$ specifies all positional and orientational degrees of freedom of a given molecule and $g_{2}\left(\mathbf{x}_{1}, \mathbf{x}_{2}\right)$ is the full pair correlation function which is related to the two-body distribution function $f_{2}\left(\mathbf{x}_{1}, \mathbf{x}_{2}\right)=f_{1}\left(\mathbf{x}_{1}\right) f_{1}\left(\mathbf{x}_{1}\right)\left[1+g_{2}\left(\mathbf{x}_{1}, \mathbf{x}_{2}\right)\right]$.

The Ornstein-Zernike equation can readily be generalised to a multicomponent system including block copolymers. In particular the direct correlation functions of rod-coil diblock copolymers are defined by the equation:

$$
\begin{aligned}
& g_{v, \eta}\left(\mathbf{r}_{1}, \mathbf{r}_{2}, \mathbf{a}_{1}, \mathbf{a}_{2}\right)=C_{v, \eta}\left(\mathbf{r}_{1}, \mathbf{r}_{2}, \mathbf{a}_{1}, \mathbf{a}_{2}\right)+ \\
& \int C_{v, \gamma}\left(\mathbf{r}_{1}, \mathbf{r}_{3}, \mathbf{a}_{1}, \mathbf{a}_{3}\right) g_{\gamma, \eta}\left(\mathbf{r}_{2}, \mathbf{r}_{3}, \mathbf{a}_{2}, \mathbf{a}_{3}\right) \rho_{\eta}\left(\mathbf{r}_{3}, \mathbf{a}_{3}\right) d \mathbf{r}_{3} d \mathbf{a}_{3}
\end{aligned}
$$

where $(v, \eta)=(r, c)$.

In the isotropic phase, $\rho_{\eta}(\mathbf{r}, \mathbf{a})=$ const and hence the Fourier transforms of the direct correlation functions of rod- 
coil block copolymers satisfy the following matrix equation:

$$
g_{v, \eta}(\mathbf{q}, \mathbf{a})=C_{v, \eta}^{0}(\mathbf{q}, \mathbf{a})+\rho_{\gamma} g_{v, \gamma}(\mathbf{q}, \mathbf{a}) C_{\gamma, \eta}^{0}(\mathbf{q}, \mathbf{a}),
$$

where all correlation functions depend also on the unit vector a.

This equation can be solved in the matrix form to obtain the following explicit expression for the direct correlation functions:

$$
\begin{aligned}
C_{v, \gamma}^{0}(\mathbf{q}, \mathbf{a}) & =g_{v, \eta}(\mathbf{q}, \mathbf{a})\left[\delta_{\gamma, \eta}+\rho_{\gamma} g_{\gamma, \eta}(\mathbf{q}, \mathbf{a})\right]^{-1} \\
& =\rho_{v}^{-1} \delta_{v, \gamma}-\left[\rho_{v} \delta_{v, \gamma}+\rho_{v} \rho_{\gamma} g_{v, \gamma}(\mathbf{q}, \mathbf{a})\right]^{-1}
\end{aligned}
$$

The density-density correlation function of rod-coil block copolymers is given by the following general equation:

$$
G_{v, \gamma}\left(\mathbf{x}_{1}, \mathbf{x}_{2}\right)=\left\langle\delta \rho_{v}\left(\mathbf{x}_{1}\right) \delta \rho_{\gamma}\left(\mathbf{x}_{2}\right)\right\rangle
$$

where $\delta \rho_{v}(\mathbf{x})=\rho_{v}^{M}(\mathbf{x})-\rho_{v}$. Here $\rho_{v}$ is the average number density of the component $v$ and $\rho_{v}^{M}(\mathbf{x})$ is the microscopic number density which is expressed as:

$$
\rho_{v}^{M}(\mathbf{x})=\sum_{i} \delta\left(\mathbf{x}-\mathbf{x}_{i}\right)
$$

where $\mathbf{x}_{i}$ specifies the coordinates of the molecule $i$.

In the isotropic phase $\left\langle\rho_{v}^{M}(\mathbf{x})\right\rangle=\rho_{v}$ and hence the densitydensity correlation function between the monomers within one macromolecule in the isotropic phase can be written in the form:

$$
G_{\nu, \gamma}\left(\mathbf{r}_{12}, \mathbf{a}\right)=\rho_{\nu} \delta_{v, \gamma} \delta\left(\mathbf{r}_{12}\right)+\rho_{\nu} \rho_{\gamma} g_{v, \gamma}\left(\mathbf{r}_{12}, \mathbf{a}\right) .
$$

Using Eqs. (15) and (18), the direct correlation functions $C_{v, \gamma}^{0}(\mathbf{q}, \mathbf{a})$ can be expressed in terms of the density-density correlation functions $G_{v, \gamma}\left(\mathbf{r}_{12}, \mathbf{a}\right)$ :

$$
C_{v, \gamma}^{0}(\mathbf{q}, \mathbf{a})=\rho_{v}^{-1} \delta_{v, \gamma}-G_{v, \gamma}(\mathbf{q}, \mathbf{a})^{-1}
$$

The coil-coil density-density correlation function for block copolymers has been calculated by several authors ${ }^{20,60,61}$ starting from the classical paper by Leibler ${ }^{20}$ using the approximation of long Gaussian chains:

$$
\begin{aligned}
G_{c c}(q)=\rho_{0} \frac{1}{N} \int_{0}^{N f_{c}} & \int_{0}^{s} \exp \left[\left(s-s^{\prime}\right) q^{2} a^{2} / 6\right] d s d s^{\prime} \\
& =\rho_{0} N \frac{2}{x^{2}}\left(f_{c} x+\exp \left(-f_{c} x\right)-1\right)
\end{aligned}
$$

where $N$ is the total number of segments in the polymer molecule, $f_{c}$ is the fraction of coil segments, $a$ is the radius of the monomer, $x=q^{2} N a^{2} / 6=q^{2} R^{2}$ and it has been assumed that $f_{c} N \gg 1$.

The rod-coil and rod-rod density correlation functions for diblock copolymers, averaged over all orientations of the rod segment, have been calculated in the classical paper ${ }^{19}$. The corresponding non-averaged correlation functions $G_{r c}(\mathbf{q}, \mathbf{a})$ and $G_{r r}(\mathbf{q}, \mathbf{a})$ which depend on the orientation of the rod segment of a diblock molecule can be calculated using general expressions derived in ${ }^{19}$. In particular, the rod-coil correlation function can be expressed as:

$$
G_{r c}(\mathbf{q}, \mathbf{a})=\rho_{0} N f_{r} f_{c} K_{R}^{(1)}(y) K_{c}^{(1)}(x),
$$

where

$$
K_{c}^{(1)}(x)=\frac{1}{x}[1-\exp (-x)]
$$

and

$$
K_{R}^{(1)}(y)=\operatorname{Re} \frac{1}{N f_{r}} \int_{0}^{N f_{r}} \exp [i(\mathbf{q} \cdot \mathbf{a}) s] d s=\frac{\sin (y)}{y},
$$

where $y=N f_{r} q a(\mathbf{k} \cdot \mathbf{a})$ and where $f_{r}$ is the fraction of rod segments.

The rod-rod correlation function can be written in the form:

$$
\begin{array}{r}
G_{r r}(\mathbf{q}, \mathbf{a})= \\
\rho_{0} \frac{1}{N} \int_{0}^{N f_{r}} \int_{0}^{N f_{r}} \exp \left[i\left(s-s^{\prime}\right) q a(\mathbf{q} \cdot \mathbf{a})\right] d s d s^{\prime}= \\
2 \rho_{0} N f_{r}^{2} \frac{(1-\cos y)}{y^{2}} .
\end{array}
$$

One notes that rod-rod and rod-coil density correlation functions depend on the orientation of the unit vector a along the rod segment with respect to the wave vector q. This orientational dependence is rather complicated and in the first approximation it is possible to expand the correlation functions in Legendre polynomials $P_{2 n}((\mathbf{k} \cdot \mathbf{a}))$ keeping the first few term:

$$
C_{r c}(\mathbf{q}, \mathbf{a}) \approx C_{r c}^{0}(q)+C_{r c}^{(2)}(q) P_{2}(\mathbf{a} \cdot \mathbf{k}),
$$

where $\mathbf{k}=\mathbf{q} / q$. In a similar way, the rod-rod correlation function is approximately given by:

$$
\begin{aligned}
C_{r r}\left(\mathbf{q}, \mathbf{a}_{1}, \mathbf{a}_{2}\right) \approx C_{c r}^{0}(q)+ & \frac{1}{2} C_{r r}^{(2)}(q) P_{2}\left(\mathbf{a}_{1} \cdot \mathbf{k}\right) \\
& +\frac{1}{2} C_{r r}^{(2)}(q) P_{2}\left(\mathbf{a}_{2} \cdot \mathbf{k}\right),
\end{aligned}
$$

where we have taken into account that the correlation function is symmetric. After substitution of the rod-rod correlation function into the mean-field potentials, one should set $\mathbf{a}_{1}=\mathbf{a}_{2}=\mathbf{a}$ as the rod segments within one macromolecule are always parallel.

\section{TILTED LAMELLAR PHASE}

In the orthogonal lamellar phase the one-particle densities should be periodic functions with the period of the phase. Hence all "mean-field" potentials, i.e., all terms in the exponential functions in Eqs. (11) and (10), are also periodic with the same period and can be expanded in Fourier series. Thus in the first approximation the corresponding terms can be approximated by the following first terms of the Fourier expansion:

$$
\int C_{c c}\left(\mathbf{r}_{12}\right) \delta \rho_{c}\left(\mathbf{r}_{2}\right) d \mathbf{r}_{2}=\rho_{0} f_{c} \psi_{c} C_{c c}(q) \cos \left(\mathbf{q}_{i} \cdot \mathbf{r}_{1}\right)
$$




$$
\begin{gathered}
\int C_{r r}\left(\mathbf{r}_{12}, \mathbf{a}\right) \boldsymbol{\delta} \rho_{r}\left(\mathbf{r}_{2}, \mathbf{a}\right) d \mathbf{r}_{2}= \\
\rho_{0} f_{r} \psi_{r}\left[C_{r r}^{(0)}(q) \cos \left(\mathbf{q}_{i} \cdot \mathbf{r}_{1}\right)\right. \\
\left.+\frac{1}{2} C_{r r}^{(2)}(q) P_{2}\left(\mathbf{a} \cdot \mathbf{k}_{i}\right) \cos \left(\mathbf{q} \cdot \mathbf{r}_{1}\right)\right] \\
+\frac{1}{2} \rho_{0} f_{r} \sigma C_{r r}^{(2)}(q) \cos \left(\mathbf{q} \cdot \mathbf{r}_{1}\right) \\
\int C_{c r}\left(\mathbf{r}_{12}, \mathbf{a}\right) \delta \rho_{c}\left(\mathbf{r}_{2}\right) d \mathbf{r}_{2} \\
=\rho_{0} f_{c} \psi_{c}\left[C_{c r}^{(0)}(q) \cos (\mathbf{q} \cdot \mathbf{k})\right. \\
\left.+C_{c r}^{(2)}(q) P_{2}\left(\mathbf{a} \cdot \mathbf{r}_{1}\right) \cos \left(\mathbf{q}_{i} \cdot \mathbf{r}_{1}\right)\right] \\
\int C_{c r}\left(\mathbf{r}_{12}, \mathbf{a}\right) \delta \rho_{r}\left(\mathbf{r}_{2}, \mathbf{a}\right) d \mathbf{r}_{2} d \mathbf{a} \\
=\rho_{0} f_{r} \cos \left(\mathbf{q} \cdot \mathbf{r}_{1}\right)\left[\psi_{r} C_{c r}^{(0)}(q)+\sigma C_{c r}^{(2)}(q)\right]
\end{gathered}
$$

and

$$
\begin{array}{r}
\int J\left(\mathbf{r}_{12}\right) P_{2}\left(\mathbf{a}_{1} \cdot \mathbf{a}_{2}\right) \delta \rho_{r}\left(\mathbf{r}_{2}, \mathbf{a}_{2}\right) d \mathbf{r}_{2} d \mathbf{a}_{2} \\
=\rho_{0} f_{r}\left\{J_{0} S P_{2}(\mathbf{a} \cdot \mathbf{n})+J_{1} \gamma P_{2}(\mathbf{a} \cdot \mathbf{n}) \cos \left(\mathbf{q} \cdot \mathbf{r}_{1}\right)\right. \\
\left.+J_{2} \sigma P_{2}(\mathbf{a} \cdot \mathbf{n}) \cos \left(\mathbf{q} \cdot \mathbf{r}_{1}\right)\right\} .
\end{array}
$$

Here $\mathbf{k}$ is the unit vector along $\mathbf{q}$ and the order parameters are defined by the following expressions:

$$
\begin{gathered}
\psi_{r}=\left\langle\cos \left(\mathbf{q}_{i} \cdot \mathbf{r}\right)\right\rangle=\int \delta \rho_{r}(\mathbf{r}, \mathbf{a}) \cos (\mathbf{q} \cdot \mathbf{r}) d \mathbf{r} d \mathbf{a}, \\
\psi_{c}=\left\langle\cos \left(\mathbf{q}_{i} \cdot \mathbf{r}\right)\right\rangle=\int \delta \rho_{c}(\mathbf{r}) \cos (\mathbf{q} \cdot \mathbf{r}) d \mathbf{r}, \\
\sigma_{k}=\left\langle P_{2}(\mathbf{a} \cdot \mathbf{k}) \cos (\mathbf{q} \cdot \mathbf{r})\right\rangle, \\
S=\left\langle P_{2}(\mathbf{a} \cdot \mathbf{n})\right\rangle ; \sigma=\left\langle P_{2}(\mathbf{a} \cdot \mathbf{n}) \cos (\mathbf{q} \cdot \mathbf{r})\right\rangle,
\end{gathered}
$$

where we have set $\mathbf{a}_{1}=\mathbf{a}_{2}=\mathbf{a}$ in the final expressions because all rod segments are always parallel within one macromolecule.

Finally the equilibrium densities of rod and coil monomers in the tilted lamellar phase can be explicitly expressed in terms of the translational and orientational order parameters (32-35) using Eqs. (27-31):

$$
\begin{aligned}
\rho_{c}(\mathbf{r})=Z_{c}^{-1} \exp \left\{\rho_{0} \cos (\mathbf{q} \cdot \mathbf{r})\right. & \\
\times\left[f_{c} C_{c c}^{(0)}(q)\right. & \psi_{c}+f_{r} C_{c r}^{(0)}(q) \psi_{r} \\
& \left.\left.+f_{r} C_{c r}^{(2)}(q) \sigma_{k}-f_{r} \chi \psi_{r}\right]\right\}
\end{aligned}
$$

$$
\begin{aligned}
& \rho_{r}(\mathbf{r}, \mathbf{a})= Z_{r}^{-1} \exp \left\{\rho_{0} \cos (\mathbf{q} \cdot \mathbf{r})\right. \\
& \times {\left[f_{r} C_{r r}^{(0)}(q) \psi_{r}+f_{c} C_{c r}^{(0)}(q) \psi_{c}-f_{c} \chi \psi_{c}\right]+} \\
&+\rho_{0} P_{2}(\mathbf{a} \cdot \mathbf{k}) \cos (\mathbf{q} \cdot \mathbf{r})\left[f_{c} C_{c r}^{(2)}(q) \psi_{c}\right. \\
&+\left.\frac{1}{2} f_{r} C_{r r}^{(2)}(q) \psi_{r}\right]+\rho_{0} P_{2}(\mathbf{a} \cdot \mathbf{n}) \cos (\mathbf{q} \cdot \mathbf{r}) f_{r} \beta J_{2} \sigma+ \\
&\left.\frac{1}{2} \rho_{0} C_{r r}^{(2)}(q) f_{r} \sigma_{k} \cos (\mathbf{q} \cdot \mathbf{r})+\rho_{0} f_{r} \beta J_{0} S P_{2}(\mathbf{a} \cdot \mathbf{n})\right\}
\end{aligned}
$$

It should be noted that in the tilted lamellar phase the director $\mathbf{n}$ is not parallel to the wave vector $\mathbf{q}$. As a result the order parameter $\sigma_{k}=\left\langle P_{2}(\mathbf{a} \cdot \mathbf{k}) \cos (\mathbf{q} \cdot \mathbf{r})\right\rangle$ is different from the order parameter $\sigma=\left\langle P_{2}(\mathbf{a} \cdot \mathbf{k}) \cos (\mathbf{n} \cdot \mathbf{r})\right\rangle$ because in the expression for $\sigma$ the long molecular axis $\mathbf{a}$ is coupled with the director $\mathbf{n}$ while in the expression for $\sigma_{k}$ it is coupled with $\mathbf{q}$.

Similarly to the orientational tensor order parameter (2), the tensor order parameter describing the simultaneous orientational and translational order can be presented as ${ }^{62}$ :

$$
\Sigma_{i j}=\sigma\left(n_{i} n_{j}-\frac{1}{3} \delta_{i j}\right)+\Pi\left(m_{i} m_{j}-h_{i} h_{j}\right),
$$

where $\Pi$ are the corresponding biaxial order parameter. Here the unit vectors $\mathbf{n}, \mathbf{m}$ and $\mathbf{h}$ are the same primary axes as those of the tensor $Q_{i j}$. In particular, $\mathbf{n}$ is the director and $\mathbf{l}$ is parallel to the $C_{2}$ symmetry axis of the $\mathrm{SmC}$ phase which is normal to the tilt plane. Thus the orientational-translational order in the tilted phase can be described by three scalar order parameters: $\sigma, \Pi$ and the tilt angle $\theta$ between the director $\mathbf{n}$ and the wave vector $\mathbf{q}$ while the orientational order is described by the scalar order parameters $S, P$ and the tilt angle $\theta$.

Also similarly to the tensor order parameters $Q_{i j}$ in the form (3), $\Sigma_{i j}$ can be expressed in the Cartesian frame spanned by the layer normal $\mathbf{k}$, unit vector $\mathbf{h}$ and the unit vector $\mathbf{c}$ which is parallel to the lamellar plane and perpendicular to $\mathbf{h}$ as:

$\Sigma_{i j}=\Sigma_{k}\left(k_{i} k_{j}-\delta_{i j} / 3\right)+\frac{1}{2} \Pi_{k}\left(c_{i} c_{j}-h_{i} h_{j}\right)+\frac{1}{2} \sigma_{\theta}\left(k_{i} c_{j}+c_{i} k_{j}\right)$,

where $\Sigma_{k}, \Pi_{k}, \sigma_{\theta}$ are the corresponding orientationaltranslational order parameters of the tilted lamellar phase which can be explicitly expressed as the following statistical averages of the corresponding molecular quantities (compare to Eqs. (4) and (5)):

$$
\begin{array}{r}
\sigma_{k}=\left\langle P_{2}(\cos \gamma) \cos (\mathbf{q} \cdot \mathbf{r})\right\rangle, \\
\Pi_{k}=\left\langle\sin ^{2} \gamma \cos 2 \varphi \cos (\mathbf{q} \cdot \mathbf{r})\right\rangle, \\
\sigma_{\theta}=\langle\sin 2 \gamma \cos \varphi \cos (\mathbf{q} \cdot \mathbf{r})\rangle,
\end{array}
$$

Here the order parameter $\sigma_{k}$ characterizes the orientational and translational order with respect to the smectic layer normal $\mathbf{k}$ while the order parameter $\Pi_{k}$ describes the biaxial distribution of long molecular axes in the lamellar plane. Finally, the tilt order parameter $\sigma_{\theta}$ in the non-diagonal term in Eq. (39) describes the tilt of the director with respect to the layer normal $\mathbf{k}$. In the orthogonal lamellar phase $\Pi_{k}=0$ and the tensor (39) becomes diagonal. An important advantage of the representations (3) and (39) of the tensor order parameters is that 
the tilt order parameters $V$ and $\sigma_{\theta}$ are explicitly expressed as statistical averages of molecular quantities while the tilt angle $\theta$ itself cannot be expressed in this way.

The order parameters $V$ and $\sigma_{\theta}$ can be analytically expressed in terms of $S, P$ and $\theta$ and $\sigma, \Pi$ and the $\theta$, respectively ${ }^{38,62}$. In particular,

$$
\tan 2 \Theta=\frac{\sigma_{\theta}}{\sigma_{k}-\frac{1}{2} \Pi_{k}}
$$

and a similar expression is also valid for $V$. Thus one concludes that the tilt order parameters $V$ and $\sigma_{\theta}$ are linear in $\theta$ at small tilt angle $\theta$. Taking into account that $S^{2}=S_{k}^{2}+\frac{3}{4} P_{k}^{2}+$ $\frac{3}{4} V^{2}$ and $\sigma^{2}=\sigma_{k}^{2}+\frac{3}{4} \Pi_{k}^{2}+\frac{3}{4} \sigma_{\theta}^{238,62}$, one obtains the following expression for the free energy:

$$
\begin{aligned}
\frac{\beta \Delta F}{V}=\frac{1}{2} \rho_{0}^{2}[ & C_{c c}^{(0)}(q) \psi_{c}^{2} f_{c}^{2}+2 C_{c r}^{(0)}(q) \psi_{c} \psi_{r} f_{c} f_{r}+C_{r r}^{(0)}(q) \psi_{r}^{2} f_{r}^{2}+2 C_{c r}^{(2)}(q) \psi_{c} \sigma_{k} f_{c} f_{r}+C_{r r}^{(2)}(q) \psi_{r} \sigma_{k} f_{r}^{2}- \\
& \left.-2 \chi \psi_{c} \psi_{r} f_{c} f_{r}+\beta J_{2}\left(\sigma_{k}^{2}+\frac{3}{4} \Pi_{k}^{2}+\frac{3}{4} \sigma_{\theta}^{2}\right) f_{r}^{2}\right]+\frac{1}{2} \rho_{0}^{2} f_{r}^{2} \beta J_{0}\left(S_{k}^{2}+\frac{3}{4} P_{k}^{2}+\frac{3}{4} V^{2}\right)-\rho_{0}\left(f_{c} \ln Z_{c}+f_{r} \ln Z_{r}\right),
\end{aligned}
$$

where the partition function $Z_{r}$ is expressed as:

$$
\begin{aligned}
& Z_{r}=\int d \mathbf{r} d \mathbf{a} \exp \left\{\rho_{0} \cos (\mathbf{q} \cdot \mathbf{r})\right. \\
& \times\left[f_{r} C_{r r}^{(0)}(q) \psi_{r}+f_{c} C_{c r}^{(0)}(q) \psi_{c}-f_{c} \chi \psi_{c}\right]+ \\
& \quad+\rho_{0} P_{2}(\mathbf{a} \cdot \mathbf{k}) \cos (\mathbf{q} \cdot \mathbf{r}) \\
& \times\left[f_{c} C_{c r}^{(2)}(q) \psi_{c}+\frac{1}{2} f_{r} C_{r r}^{(2)}(q) \psi_{r}\right] \\
&+\frac{1}{2} \rho_{0} C_{r r}^{(2)}(q) f_{r} \sigma_{k} \cos (\mathbf{q} \cdot \mathbf{r}) \\
&+\rho_{0} f_{r} \beta J_{2} \cos (\mathbf{q} \cdot \mathbf{r}) \sigma P_{2}(\mathbf{a} \cdot \mathbf{n}) \\
&\left.\quad+\rho_{0} f_{r} \beta J_{0} S P_{2}(\mathbf{a} \cdot \mathbf{n})\right\}
\end{aligned}
$$

Using the following useful relations:

$$
\begin{aligned}
S P_{2}(\mathbf{a} \cdot \mathbf{n})= & \frac{3}{2}\left(a_{i} a_{j}-\frac{1}{3} \delta_{i j}\right) Q_{i j}= \\
= & S_{k} P_{2}(\mathbf{a} \cdot \mathbf{k})+\frac{3}{2} V(\mathbf{a} \cdot \mathbf{c})(\mathbf{a} \cdot \mathbf{k}) \\
& =S_{k} P_{2}(\mathbf{a} \cdot \mathbf{k})+\frac{3}{4} V \sin 2 \gamma \cos \varphi \\
& \\
\sigma P_{2}(\mathbf{a} \cdot \mathbf{n})= & \sigma_{k} P_{2}(\mathbf{a} \cdot \mathbf{k})+\frac{3}{4} \sigma_{\theta} \sin 2 \gamma \cos \varphi
\end{aligned}
$$

one can expand the free energy of the tilted lamellar phase in powers of $\theta$ presuming that the parameters $V$ and $\sigma_{\theta}$ are linear in $\theta$ while the biaxial order parameters $P_{k}$ and $\Pi_{k}$ are of the higher order in $\theta$ :

$$
\frac{\beta F_{c}}{V}=\frac{\beta F_{a}}{V}+\alpha_{1} V^{2}+2 \alpha_{2} V \sigma_{\theta}+\alpha_{3} \sigma_{\theta}^{2},
$$

where

$$
\alpha_{1}=\frac{3 \beta J_{0}}{8} \rho_{0}^{2} f_{r}^{2}-\left(\frac{3 \beta J_{0}}{8}\right)^{2} \rho_{0}^{3} f_{r}^{3}\left\langle\sin ^{2} 2 \gamma\right\rangle_{0}
$$

$$
\begin{gathered}
\alpha_{2}=-\frac{9 \beta^{2} J_{0} J_{2}}{64} \rho_{0}^{3} f_{r}^{3}\left\langle\sin ^{2} 2 \gamma \cos (\mathbf{q} \cdot \mathbf{r})\right\rangle_{0}, \\
\alpha_{3}=\frac{3 \beta J_{2}}{8} \rho_{0}^{2} f_{r}^{2}-\left(\frac{3 \beta J_{2}}{8}\right)^{2} \rho_{0}^{3} f_{r}^{3}\left\langle\sin ^{2} 2 \gamma \cos ^{2}(\mathbf{q} \cdot \mathbf{r})\right\rangle_{0} .
\end{gathered}
$$

where the averages

$$
\langle\ldots\rangle_{0}=\int \ldots \rho_{a}(\mathbf{r}, \mathbf{a}) d \mathbf{r} d \mathbf{a},
$$

are taken with the distribution function of rods in the orthogonal lamellar phase:

$$
\begin{aligned}
& \rho_{a}(\mathbf{r}, \mathbf{a})=\frac{1}{Z_{a}} \exp \left\{\rho_{0} \cos (\mathbf{q} \cdot \mathbf{r})\right. \\
& \times\left[f_{r} C_{r r}^{(0)}(q) \psi_{r}+f_{c} C_{r c}^{(0)}(q) \psi_{c}-f_{c} \chi \psi_{c}\right]+ \\
& \quad+\rho_{0} P_{2}(\mathbf{a} \cdot \mathbf{k}) \cos (\mathbf{q} \cdot \mathbf{r}) \times \\
& {\left[f_{c} C_{r c}^{(2)}(q) \psi_{c}+\frac{1}{2} f_{r} C_{r r}^{(2)}(q) \psi_{r}\right]+\rho_{0} \beta J_{2} \cos (\mathbf{q} \cdot \mathbf{r}) P_{2}(\mathbf{a} \cdot \mathbf{k}) \sigma+} \\
& \left.\frac{1}{2} \rho_{0} C_{r r}^{(2)}(q) f_{r} \sigma \cos (\mathbf{q} \cdot \mathbf{r})+\rho_{0} f_{r} \beta J_{0} S P_{2}(\mathbf{a} \cdot \mathbf{k})\right\} d \mathbf{r} d \mathbf{a}, \quad \text { (53) }
\end{aligned}
$$

and

$$
Z_{a}=\int \rho_{a}(\mathbf{r}, \mathbf{a}) d \mathbf{r} d \mathbf{a}
$$

Note that $\rho_{a}$ is independent of $\varphi$, so the averaging over $\varphi$ reduces to multiplying by $1 / 2$.

The free energy (48) of the lamellar phase is now expressed as a quadratic form in terms of the two tilt order parameters $V \ll 1$ and $\sigma_{\theta} \ll 1$ and the stability of the orthogonal lamellar phase is determined by the coefficients $\alpha_{1}, \alpha_{2}$ and $\alpha_{3}$ of this form. The orthogonal phase is stable if $\alpha_{1}>0, \alpha_{3}>0$ and $\alpha_{2}^{2}-\alpha_{1} \alpha_{3}<0$. The instability with respect to the tilting occurs when one of the coefficients $\alpha_{1}$ or $\alpha_{3}$ or both of them are negative. Also a strong coupling determined by the coefficient 

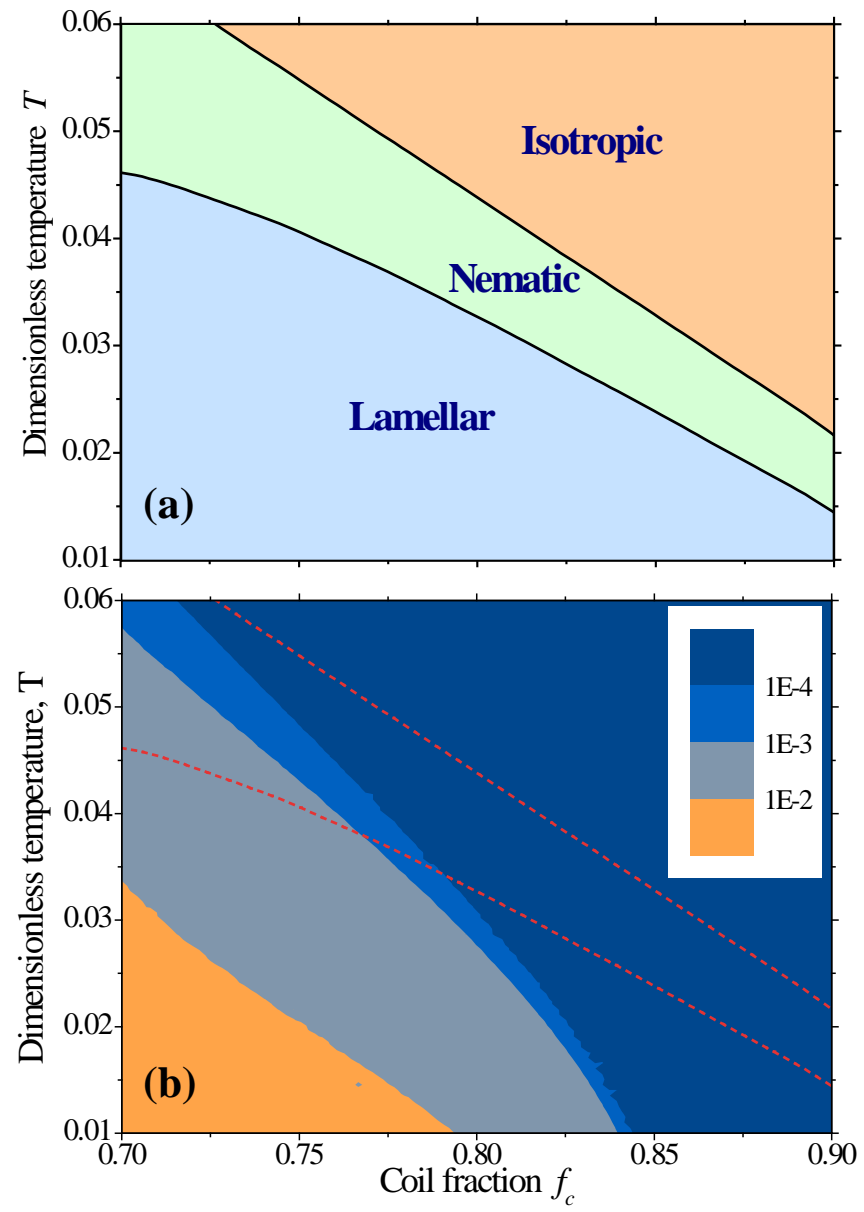

FIG. 6. Phase diagram (a) and logarithmic colourmap of the determinant $\alpha_{2}^{2}-\alpha_{1} \alpha_{3}(\mathrm{~b})$ in the axes of the coil fraction $f_{c}$ and dimensionless temperature $T=\left(\beta J_{0}\right)^{-1}$ numerically calculated for $N=20$, $\chi=2$, and $J_{2}=J_{0} / 3$. Three present phases are indicated in (a), the phase transition lines are shown by the dashed lines in (b).

$\alpha_{2}$ creates instability when $\alpha_{2}^{2}-\alpha_{1} \alpha_{3}<0$ even if $\alpha_{1}$ and $\alpha_{3}$ both stay positive.

To illustrate DFT predictions for the copolymer with the parameters close to those in the above computer simulations, we study the particular case of the phase diagram presented in Fig. 6(a), which is obtained similarly to those in Ref. ${ }^{42}$. Resolving numerically the values of all three parameters $\alpha_{1,2,3}$ we observe that over the whole diagram the coefficients $\alpha_{1}$ and $\alpha_{3}$ are positive reaching the values 0.7-0.8 in the bottom left corner. However, the coefficient $\alpha_{2}$ of the cross term in Eq. (48) is also large and exceeds them both. As a result, as shown in Fig. 6(b), $\alpha_{2}^{2}-\alpha_{1} \alpha_{3}>0$ over the whole diagram, and the tilting is thermodynamically preferable. This means that although the orthogonal lamellar phase is generally stable against a separate tilt of the orientational-density wave specified by the order parameter $\sigma_{\theta}$ as well as against a tilt of the director in an individual lamella described by $V$, strong coupling of these parameters gives rise to an instability. As also follows from Fig. 6(b), there is a considerable range of the diagram especially at higher $f_{c}$ where the instability is subtle: the cross term constant only marginally exceeds its critical value. At the same time, at lower coil fraction $f_{c}$ and lower temperature the instability becomes much more pronounced. This enables one to interpret the computer simulation results presented in Section II. Extrapolating the phase diagram obviously suggests that at $f_{c} \approx 0.5$ (the value used in simulations) the orthogonal lamellar phase is generally unstable at all reasonable temperatures which corresponds to the results of simulations. One notes that the temperature is not present explicitly in the simulations but it is well known ${ }^{63}$ that the parameter $\chi$ which specifies the repulsion between rod and coil monomers is effectively temperature dependent i.e. $\chi=\chi_{1}+\chi_{2} / T$. The simulations have been performed in the broad range of the segregation parameter $\chi N$ between 0 and 200 which effectively corresponds to a rather broad temperature range.

\section{DISCUSSION}

In this paper we have performed DPD simulations of the tilted lamellar phase exhibited by rod-coil diblock copolymers and developed a molecular -statistical theory which enables one to describe the limit of stability of the orthogonal lamellar phase with respect to the tilt of the director which specifies the direction of the orientational ordering of the rod fragments. In computer simulations the copolymer molecule has been modelled by a string of 20 interaction cites with approximately half of them representing the flexible chain and another half representing the rigid rod. the DPD simulations have been undertaken in a broad range of the segregation parameter $\chi N$, where the Flory-Higghins parameter $\chi$ specifies the incompatibility of rod and coil monomers. It has been found that for all values of the parameters investigated the only stable phase is the tilted lamellar phase. In this phase the rods are strongly orientationally ordered and are collectively tilted away from the layer normal. At the same time the degree of the orientational order and the value of the tilt increase with the increasing segregation parameter and are very sensitive to the fraction of the rod monomers. The orientational order parameters and the values of the tilt angle have been extracted from the simulation data by direct diagonalization of the ordering tensor and by using the rigorous expression for the tilt angle in terms of the uniaxial and biaxial orientational order parameters of the tilted phase derived in ${ }^{38}$. A good agreement between the results have been found.

The general density functional theory of rod-coil diblock copolymers developed by the authors before ${ }^{42}$ has been generalised to the case of the tilted lamellar phase. One notes that such a density functional theory is based on the selfconsistent equations for the equilibrium densities of rod and coil monomers and for the corresponding orientational and translational order parameters and hence it cam be used both in the case of both weak and relatively strong segregation. An explicit expression for the free energy of the tilted lamellar phase has been obtained which depends on a number of additional order parameters which specify the orientational and 
translational ordering in the low symmetry tilted phase. Indeed, in the orthogonal uniaxial phase the orientational order of rods is described by the single orientational order parameter $S$ and the simultaneous orientational-translational ordering is described by the only order parameter $\sigma$. In contrast, the tilted lamellar phase is biaxial and the primary axis of any ordering tensor is tilted with respect to the layer normal. As a result the orientational order is specified by three order parameters $<$ including the biaxiality parameter and the order parameter $V$ which accounts for the tilt of the primary axis of the ordering tensor. The same is valid for the description of the orientational-translational order which results in another tilt order parameter $\sigma_{\theta}^{38,62}$. One notes that these order parameters have not been employed in the existing theory of block copolymers. Thus the tilting transition in the lamellar phase is characterised by two tilt order parameters and the stability of the orthogonal phase is determined by the coefficients of the corresponding quadratic terms in the free energy expansion in powers of $V$ and $\sigma_{\theta}$.

The stability limit of the orthogonal lamellar phase has been investigated by numerical calculations of the corresponding coefficients in the quadratic terms in the free energy expansion in terms of the two tilt order parameters as functions of temperature and the copolymer composition. It has been found that that the temperature range, in which the orthogonal phase is unstable, is increasing rapidly with the decreasing coil fraction $f_{c}$. This enables one to conclude that in the range of parameters used in simulations) the orthogonal lamellar phase is generally unstable which is consistent with the results of the simulations. One notes that the Flory-Higgins parameter $\chi$ is effectively temperature dependent i.e. $\chi=\chi_{1}+\chi_{2} / T^{14}$. Taking into account that the simulations have been performed in the broad range of the segregation parameter $\chi N$ one concludes that a rather broad temperature range has been effectively covered.

Finally one notes that the present theory in principle enables one to calculate numerically the tilt angle in the tilted lamellar phase by direct minimization of the free energy. This procedure requires a numerical minimization with respect to eight orientational and translation order parameters, and it will be implemented in our next publication.

\section{ACKNOWLEDGMENTS}

M.A.O., M.V.G., A.K.B. and A.A.A. are grateful to Russian Science Foundation, Grant 19-13-00398 for financial support.

\section{Appendix A: Correlation functions of noninteracting rod-coil copolymer chains}

The coil-coil density-density correlation function for the reference disordered phase of block copolymers are presented in Section III (see Eqs. (20-23).

The direct correlation functions $C_{v \mu}$ of the disordered reference phase of noninteracting chains are given by the general Eq. (19) derived above. In particular, it yields the following explicit expressions:

$$
\begin{gathered}
C_{r r}^{0}(\mathbf{q}, \mathbf{a})=\frac{1}{\rho_{0} f_{r}}-\frac{G_{c c}^{0}}{D e t^{0}} \\
C_{c c}^{0}(\mathbf{q}, \mathbf{a})=\frac{1}{\rho_{0} f_{c}}-\frac{G_{r r}^{0}}{D e t^{0}} \\
C_{r c}^{0}(\mathbf{q}, \mathbf{a})=\frac{G_{r c}^{0}}{D e t^{0}},
\end{gathered}
$$

where

$$
D e t^{0}=G_{r r}^{0} G_{c c}^{0}-\left(G^{0}\right)_{r c}^{2}
$$

where

$$
\begin{aligned}
& G_{r r}^{0}(q)=\int G_{r r}(\mathbf{q}, \mathbf{a}) d \mathbf{a}, \\
& G_{c c}^{0}(q)=\int G_{c c}(\mathbf{q}, \mathbf{a}) d \mathbf{a}, \\
& G_{r c}^{0}(q)=\int G_{r c}(\mathbf{q}, \mathbf{a}) d \mathbf{a} .
\end{aligned}
$$

Finally the functions $C_{v \eta}^{2}(\mathbf{q})$ can be written in the form:

$$
\begin{aligned}
& C_{r r}^{(2)}(q)=C_{r r}^{1}(\mathbf{q})-C_{r r}^{0}(q), \\
& C_{c c}^{(2)}(q)=C_{c c}^{1}(\mathbf{q})-C_{c c}^{0}(q), \\
& C_{r c}^{(2)}(q)=C_{r c}^{1}(\mathbf{q})-C_{r c}^{0}(q),
\end{aligned}
$$

where the functions $C_{v \eta}^{1}(\mathbf{q})$ are obtained by setting $(\mathbf{a} \cdot \mathbf{k})=1$ in the equations for the functions $C_{v \eta}(\mathbf{q})$ and hence

$$
\begin{gathered}
C_{r r}^{1}(\mathbf{q})=\frac{1}{\rho_{0} f_{r}}-\frac{G_{c c}^{1}}{D e t^{1}} \\
C_{c c}^{1}(\mathbf{q})=\frac{1}{\rho_{0} f_{c}}-\frac{G_{r r}^{1}}{D e t^{1}} \\
C_{r c}^{1}(\mathbf{q})=\frac{G_{r c}^{1}}{D e t^{1}},
\end{gathered}
$$

where

$$
\operatorname{Det}^{1}=G_{r r}^{1} G_{c c}^{1}-\left(G_{r c}^{1}\right)^{2} .
$$

Here the functions $G_{r r}^{1}, G_{c c}^{1}, G_{r c}^{1}$ are given by Eqs. (20), (21) and (24) taken with $y=y^{*}=N f_{r} q^{*}$.

\footnotetext{
${ }^{1}$ R. A. Segalman, B. McCulloch, S. Kirmayer, and J. J. Urban, Macromolecules 42, 9205 (2009).

${ }^{2}$ K. Sivula, Z. T. Ball, N. Watanabe, and J. M. J. Fréchet, Advanced Materials 18, 206 (2006).

${ }^{3}$ J. C. M. van Hest, Polymer Reviews 47, 63 (2007).
} 
${ }^{4}$ H.-A. Klok, Journal of Polymer Science Part A: Polymer Chemistry 43, 1 (2004).

${ }^{5}$ K. Loos and A. H. E. Müller, Biomacromolecules 3, 368 (2002).

${ }^{6}$ D. M. Haddleton and K. Ohno, Biomacromolecules 1, 152 (2000).

${ }^{7}$ G. Yu, J. Gao, J. C. Hummelen, F. Wudl, and A. J. Heeger, Science 270, 1789 (1995).

${ }^{8}$ Y. Tao, B. McCulloch, S. Kim, and R. A. Segalman, Soft Matter 5, 4219 (2009).

${ }^{9}$ C. L. Chochos, J. K. Kallitsis, and V. G. Gregoriou, The Journal of Physical Chemistry B 109, 8755 (2005).

${ }^{10}$ S. Becker, C. Ego, A. C. Grimsdale, E. J. List, D. Marsitzky, A. Pogantsch, S. Setayesh, G. Leising, and K. Müllen, Synthetic Metals 125, 73 (2001).

${ }^{11}$ Y. Tao, B. Ma, and R. A. Segalman, Macromolecules 41, 7152 (2008).

${ }^{12}$ H. M. König, T. Gorelik, U. Kolb, and A. F. M. Kilbinger, Journal of the American Chemical Society 129, 704 (2007).

${ }^{13}$ Y. Pae and F. W. Harris, Journal of Polymer Science Part A: Polymer Chemistry 38, 4247 (2000).

${ }^{14}$ B. D. Olsen and R. A. Segalman, Materials Science and Engineering: R: Reports 62, 37 (2008).

${ }^{15}$ F. J. M. Hoeben, P. Jonkheijm, E. W. Meijer, and A. P. H. J. Schenning, Chemical Reviews 105, 1491 (2005).

${ }^{16}$ J.-H. Ryu and M. Lee, in Liquid Crystalline Functional Assemblies and Their Supramolecular Structures (Springer Berlin Heidelberg) pp. 63-98.

${ }^{17}$ A. Semenov and S. Vasilenko, JETP 63, 70 (1986).

${ }^{18}$ R. Hołyst and M. Schick, The Journal of Chemical Physics 96, 730 (1992).

${ }^{19}$ M. Reenders and G. ten Brinke, Macromolecules 35, 3266 (2002).

${ }^{20}$ L. Leibler, Macromolecules 13, 1602 (1980).

${ }^{21}$ M. W. Matsen and C. Barrett, The Journal of Chemical Physics 109, 4108 (1998).

${ }^{22}$ V. Pryamitsyn and V. Ganesan, The Journal of Chemical Physics 120, 5824 (2004).

${ }^{23}$ M. Müller and M. Schick, Macromolecules 29, 8900 (1996).

${ }^{24}$ J.-Z. Chen, C.-X. Zhang, Z.-Y. Sun, Y.-S. Zheng, and L.-J. An, The Journal of Chemical Physics 124, 104907 (2006).

${ }^{25}$ Y. A. Kriksin and P. G. Khalatur, Macromolecular Theory and Simulations 21, 382 (2012).

${ }^{26}$ D. Düchs and D. E. Sullivan, Journal of Physics: Condensed Matter 14, 12189 (2002).

${ }^{27}$ R. C. Hidalgo, D. E. Sullivan, and J. Z. Y. Chen, Journal of Physics: Condensed Matter 19, 376107 (2007).

${ }^{28}$ J. Tang, Y. Jiang, X. Zhang, D. Yan, and J. Z. Y. Chen, Macromolecules 48, 9060 (2015).

${ }^{29}$ W. Song, P. Tang, F. Qiu, Y. Yang, and A.-C. Shi, Soft Matter 7, 929 (2011).

${ }^{30}$ Y. Jiang and J. Z. Y. Chen, Physical Review Letters 110, 138305 (2013).

${ }^{31} \mathrm{~S}$. Li, Y. Jiang, and J. Z. Y. Chen, Soft Matter 10, 8932 (2014).

${ }^{32}$ Y. Cai, P. Zhang, and A.-C. Shi, Soft Matter 13, 4607 (2017).

${ }^{33}$ J. Gao, W. Song, P. Tang, and Y. Yang, Soft Matter 7, 5208 (2011).

${ }^{34}$ J. Gao, P. Tang, and Y. Yang, Soft Matter 9, 69 (2013).

${ }^{35}$ Y. Jiang and J. Z. Y. Chen, Physical Review E 88, 042603 (2013).
${ }^{36}$ Y. Singh, Physical Review A 30, 583 (1984).

${ }^{37}$ T. J. Sluckin and P. Shukla, Journal of Physics A: Mathematical and General 16, 1539 (1983).

${ }^{38}$ M. V. Gorkunov, M. A. Osipov, J. P. F. Lagerwall, and F. Giesselmann, Physical Review E 76 (2007), 10.1103/physreve.76.051706.

${ }^{39}$ L. Longa, J. Stelzer, and D. Dunmur, The Journal of Chemical Physics 109, 1555 (1998)

${ }^{40}$ M. A. Osipov, in Handbook of Liquid Crystals (Wiley-VCH Verlag GmbH, 2014) pp. 40-71.

${ }^{41}$ R. Evans, in Fundamentals of Inhomogeneous Fluids (New York: Decker, 1992).

${ }^{42}$ M. A. Osipov and M. V. Gorkunov, Physical Review E 100 (2019), 10.1103/physreve.100.042701.

${ }^{43}$ M. R. Wilson, A. B. Thomas, M. Dennison, and A. J. Masters, Soft Matter 5, 363 (2009)

${ }^{44}$ M. A. Horsch, Z. Zhang, and S. C. Glotzer, Physical Review Letters 95 (2005), 10.1103/physrevlett.95.056105.

${ }^{45}$ S. Dolezel, H. Behringer, and F. Schmid, Polymer Science Series C 55, 70 (2013).

${ }^{46}$ A. AlSunaidi, W. K. den Otter, and J. H. R. Clarke, Philosophical Transactions of the Royal Society of London. Series A: Mathematical, Physical and Engineering Sciences 362, 1773 (2004).

${ }^{47}$ M. A. Horsch, Z. Zhang, and S. C. Glotzer, Soft Matter 6, 945 (2010).

${ }^{48}$ A. N. Semenov, Molecular Crystals and Liquid Crystals 209, 191 (1991).

${ }^{49}$ A. A. Gavrilov, Y. V. Kudryavtsev, and A. V. Chertovich, The Journal of Chemical Physics 139, 224901 (2013).

${ }^{50}$ P. J. Hoogerbrugge and J. M. V. A. Koelman, Europhysics Letters (EPL) 19, 155 (1992).

${ }^{51}$ J. M. V. A. Koelman and P. J. Hoogerbrugge, Europhysics Letters (EPL) 21, 363 (1993).

${ }^{52}$ P. Español and P. Warren, Europhysics Letters (EPL) 30, 191 (1995).

${ }^{53}$ R. D. Groot and P. B. Warren, The Journal of Chemical Physics 107, 4423 (1997).

${ }^{54} \mathrm{http} / / /$ lammps.sandia.gov/,.

${ }^{55} \mathrm{G}$. Besold, I. Vattulainen, M. Karttunen, and J. M. Polson, Physical Review E 62, R7611 (2000).

${ }^{56}$ A. V. Berezkin, Y. V. Kudryavtsev, M. V. Gorkunov, and M. A. Osipov, The Journal of Chemical Physics 146, 144902 (2017).

${ }^{57}$ A. Polimeno, A. Gomes, and A. F. Martins, in Computer Simulations of Liquid Crystals and Polymers (Springer-Verlag) pp. 135-147.

${ }^{58}$ T. F. Miller, M. Eleftheriou, P. Pattnaik, A. Ndirango, D. Newns, and G. J. Martyna, The Journal of Chemical Physics 116, 8649 (2002).

${ }^{59}$ A. Perera, G. N. Patey, and J. J. Weis, The Journal of Chemical Physics 89, 6941 (1988).

${ }^{60}$ T. Uneyama and M. Doi, Macromolecules 38, 196 (2005).

${ }^{61}$ T. Ohta and K. Kawasaki, Macromolecules 19, 2621 (1986).

${ }^{62}$ M. Osipov and G. Pajak, Physical Review E 85 (2012), 10.1103/physreve.85.021701.

${ }^{63}$ B. D. Olsen, M. Shah, V. Ganesan, and R. A. Segalman, Macromolecules 41, 6809 (2008). 